Gynäkologe 2015 • 48:771

DOI 10.1007/s00129-015-3787-8

Online publiziert: 2. Oktober 2015

๑) Springer-Verlag Berlin Heidelberg 2015

CrossMark

\author{
K. Friese ${ }^{1} \cdot$ W. Mendling ${ }^{2}$ \\ 'Klinik Bad Trissl, Oberaudorf, Deutschland \\ ${ }^{2}$ Frauenklinik, Helios-Klinikum, Wuppertal, Deutschland
}

\title{
Infektionen in der Gynäkologie
}

Sehr geehrte Frau Kollegin,

sehr geehrter Herr Kollege,

mit diesem Themenheft möchten wir Ihnen eine aktuelle Übersicht gynäkologischer Infektionen vorstellen. Auch außerhalb unseres Fachs sind Infektionsthemen zuletzt immer wieder thematisiert worden, in der Presse, zum Teil auch in der Politik. So wie wir werden Sie bei diesem Hinweis natürlich an Ebola oder MERS denken. Gleichzeitig entsteht der Eindruck, dass in den Kliniken Infektiologie nicht mehr ausreichend gelehrt wird, wobei diese doch in unserer täglichen Arbeit eine nicht zu unterschätzende Bedeutung hat.

\section{》) Die Bedeutung der Infektiologie in der täglichen Arbeit ist nicht zu unterschätzen}

Deshalb ist es sicherlich hilfreich, wenn Herr W. Frobenius eine Bewertung, aber auch relevante Informationen zum Nativpräparat gibt, das jederzeit - wenn ein Mikroskop vorhanden ist - in Klinik und Praxis angefertigt werden kann und sehr wohl zur Differenzialdiagnostik einer möglichen Infektion oder Veränderung geeignet ist. Die von Herrn W. Mendling vorgestellte Arbeit „Vaginale Mikrobiota - ein Update“ zeigt, wie sich unsere Vorstellung vom Milieu der Scheide verändert hat und wie dies auch von außen beeinflusst werden kann.

Aus Sicht des Klinikers oder des Niedergelassenen hat man das Gefühl, dass die Salpingitis oder Adnexitis in ihrer Bedeutung abgenommen hat. Gerade deshalb ist die Arbeit von Herrn U. Hoyme sehr lesenswert, zumal er das Chlamydienscreening - aus seiner Sichtweise und die Spirale dazu in einen Kontext stellt.

Zugenommen haben in den letzten Jahren die genitalen Herpeserkrankungen, aber nicht nur HSV-2-, sondern auch die HSV1-Infektionen. Wie geht man damit um? Insbesondere: Wie kann man gezielt therapieren, wie kann man eine Rezidivprophylaxe durchführen. Hierzu informiert die Arbeit von Herrn Kollegen I. Mylonas.

In diesem Zusammenhang ist auch die Arbeit von Herrn Kollegen U. Petry und Mitarbeitern zur aktuellen Bedeutung sexuell übertragbarer Infektionen zu sehen, wenn insbesondere zur HPV-Infektion mittels der WOLVES-Studie ein genaues Bild dargestellt wird. Diese Daten gelten sicher nicht nur für den Bereich Wolfsburg, sondern lassen sich in jeden Teil Deutschlands projizieren.

\section{I) Multiresistente Keime implizieren therapeutische und finanzielle Risiken}

Abschließend weist der Artikel von Frau J. Jückstock in München auf ein immer gravierenderes und größeres Problem hin, nämlich die multiresistenten Keime, die wir in Klinik und Praxis mittlerweile beobachten und die zu einem therapeutischen, aber auch zu einem finanziellen Risiko geworden sind. Gerade hier ist es wichtig zu fragen: Wie therapieren wir, wie lange therapieren wir und welche Aussichten bestehen für neuere, aktivere Antibiotika in der Zukunft.
Wir wünschen allen geneigten Leserinnen und Lesern einen Zugewinn an Wissen.

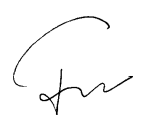

Prof. Dr. Klaus Friese

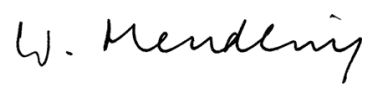

Prof. Dr. Werner Mendling

\section{Korrespondenzadresse}

Prof. Dr. K. Friese

Klinik Bad Trissl

Bad-Trissl-Str. 73, 83080 Oberaudorf,

Deutschland

klaus.friese@klinik-bad-trissl.de

Interessenkonflikt. K. Friese und W. Mendling geben an, dass kein Interessenkonflikt besteht. 